\title{
A Performance Lower Bound for Quadratic Timing Recovery Accounting for the Symbol Transition Density
}

\author{
Jaume Riba
}

\begin{abstract}
The symbol transition density in a digitally modulated signal affects the performance of practical synchronization schemes designed for timing recovery. This paper focuses on the derivation of simple performance limits for the estimation of the time delay of a noisy linearly modulated signal in the presence of various degrees of symbol correlation produced by the various transition densities in the symbol streams. The paper develops high- and low-signal-to-noise ratio (SNR) approximations of the so-called (Gaussian) unconditional Cramér-Rao bound (UCRB), as well as general expressions that are applicable in all ranges of SNR. The derived bounds are valid only for the class of quadratic, non-data-aided (NDA) timing recovery schemes. To illustrate the validity of the derived bounds, they are compared with the actual performance achieved by some well-known quadratic NDA timing recovery schemes. The impact of the symbol transition density on the classical threshold effect present in NDA timing recovery schemes is also analyzed. Previous work on performance bounds for timing recovery from various authors is generalized and unified in this contribution.
\end{abstract}

Index Terms-Conditional CRB (CCRB), conditional ML (CML), Cramér-Rao bound (CRB), Markov processes, maximum likelihood estimation (MLE), pulse shaping, self-noise, synchronization, timing jitter, transition density, unconditional CRB (UCRB), unconditional ML (UML).

\section{INTRODUCTION}

$\mathbf{T}$ HE derivation of performance bounds for determining the ultimate accuracy that can be achieved in synchronization operations is an important line of research. Parameter estimation theory offers the tools to approach this problem in the form of Cramér-Rao bounds (CRBs), which give fundamental lower limits to the variance of any estimator. In practice, the derivation of these limits in the presence of nuisance parameters is usually difficult. This happens, for instance, in the derivation of the CRBs for non-data-aided (NDA) schemes, where the data symbols themselves become the nuisance parameters. Some simplifications therefore become necessary. In this respect, the modified CRBs (MCRBs) were introduced in [1] in the general context of synchronization. These bounds are generally

Manuscript received March 21, 2003; revised October 10, 2003. This work was supported in part by the following research projects of the Spanish/Catalan Science and Technology Commisions (CICYT/CIRIT): TEC2004-04526, TIC2003-05482, TIC2002-04594, TIC2001-2356, TIC2000-1025, and 2001SGR-00268. The associate editor coordinating the review of this paper and approving it for publication was Dr. Vikram Krishnamurthy.

The author is with the Signal Processing and Communications Group, Technical University of Catalonia (UPC), 08034, Barcelona, Spain (e-mail: jriba@gps.tsc.upc.es).

Digital Object Identifier 10.1109/TSP.2004.836536 lower than the true CRBs but much simpler to compute. In fact, the simple bound obtained in [2] is actually an MCRB. In addition, mathematically simple approximations of the true CRB for timing recovery at high and low signal-to-noise ratios (SNRs) were derived in [3] and [4], respectively. In [5], the authors were able to derive a closed-form expression of the true CRB for timing recovery, but this requires some numerical integrations for its computation. A general assumption made in all these studies, however, is that the data symbols are independent.

The main advantage of NDA timing recovery schemes is their ability to extract the timing directly from the noisy signal, but it comes at the cost of requiring adequate transitions in the data symbol sequence. In this respect, the limitations of the simplifications of the CRBs mentioned above are that they lead to limits that can be very optimistic at certain SNRs, and most importantly, they are not sensitive to the actual symbol transition densities present in the received data. Low transition densities may significantly degrade the symbol timing jitter (see the analysis performed in [6] as an example), which is an important consideration to be taken into account in practice. Note, for instance, that in applications such as Earth-to-space links, the data stream may not be coded in order to simplify the equipment on board the spacecraft and that adequate transitions cannot be assured in these cases.

This paper analyzes the effect of symbol transition density on the performance limits for timing recovery of digital waveforms adequate for bandlimited channels. The study focuses on the set of quadratic timing recovery schemes, which are usually the least complex to implement in practice. Previous work dealing with effect of transition density on CRB can be found in [7], which studied the synchronizability of a class of waveforms that require the assumption of a wide-band channel for negligible distortion.

The background is introduced in Section II by establishing the so-called unconditional CRB (UCRB) derived in the context of sensor array processing [8], following the results in [9]. In Section III, the UCRB is developed for uncorrelated data, and interesting links with the work performed in [4] are found. In Section IV, we derive simple expressions for low and high SNRs in the case of correlated data that allow us to mathematically characterize the classical threshold effect observed in quadratic timing recovery schemes. In Section V, we propose a simple transition density model to establish the mathematical relationship between transition density and symbol autocorrelation. In Section VI, a general, although less 
simple, method for computing the UCRB for correlated symbols in all ranges of SNR is proposed. In Section VII, we also generalize the results for the case of a deterministic constant and alternated symbol patterns. Finally, in Section VIII, the performance of some well-known quadratic timing recovery schemes for uncorrelated and correlated data is compared with the derived bounds. The main conclusions of the paper are drawn in Section IX, along with a summary of the main results achieved.

\section{Problem Statement AND Background}

The complex baseband signal is represented as

$$
r(t)=e^{j \theta} \sum_{k=0}^{L-1} a_{k} g(t-k T-\tau)+w(t)
$$

where $\tau$ is the timing parameter to be estimated, $\theta$ is the signal phase, $\left\{a_{k}\right\}$ are zero-mean complex-valued stationary data symbols with autocorrelation $r_{a}[p]=E\left[a_{k} a_{k+p}^{*}\right]$ and pertaining to the alphabet $a_{k} \in a^{(n)}(n=0, \ldots, N-1)$, where $N$ is the alphabet size, $T$ is the symbol interval, $g(t)$ is a realvalued signalling pulse, and $w(t)$ is complex-valued zero-mean Gaussian noise with independent real and imaginary parts, each one having a power spectral density of $N_{o}$. In the above model (1), $L$ represents the number of symbols considered in the observation interval that will be used for timing estimation. The symbol energy of the modulated passband signal is defined as $E_{s}=\sigma_{a}^{2} E_{g} / 2$, where $\sigma_{a}^{2}=r_{a}[0]$. By sampling $r(t)$ at intervals $T_{s}=T / N_{\mathrm{ss}}$, where $N_{\mathrm{ss}}$ is the number of samples per symbol, and assuming that the sampling frequency $F_{s}=1 / T_{s}$ is above the Nyquist frequency, a discrete model can be used for $r(t)$ as follows [9], [10]:

$$
\mathbf{r}=\mathbf{A}_{\tau} \mathbf{x}+\mathbf{w}
$$

where $\mathbf{r}$ is the sampled signal vector, the $k$ th column of matrix $\mathbf{A}_{\tau}$ is formed by the samples of $g(t-k T-\tau)$, $\mathbf{x}=e^{j \theta}\left[a_{0}, a_{1}, \ldots, a_{L-1}\right]^{T}$ with covariance $\boldsymbol{\Gamma}$, and $\mathbf{w}$ is the noise vector of covariance $\mathbf{C}_{\mathbf{w}}=\sigma^{2} \mathbf{I}$, where $\sigma^{2}=2 N_{o} / T_{s}$. For simplicity, the paper is focused only to the case of pulses that do not generate intersymbol interference (ISI) at the matched filter output, which means that

$$
T_{s} \mathbf{A}_{\tau}^{T} \mathbf{A}_{\tau}=E_{g} \mathbf{I}
$$

where $E_{g}$ is the pulse energy.

In the context of sensor array processing (see [8], [11], and references therein), two methods for the derivation of the CRBs have been defined, which differ on how the nuisance parameters are treated. While the conditional (or deterministic) model assumes the nuisance parameters to be nonrandom (i.e., the same in all realizations), the unconditional ${ }^{1}$ (or stochastic) model assumes them to be Gaussian random processes. These two models lead to different maximum likelihood (ML) approaches, which are termed conditional ML (CML) and unconditional ML (UML). Following these two approaches,

\footnotetext{
${ }^{1}$ The word unconditional is used in the classical terminology to indicate that the statistical properties of the observed signal are unconditional with respect to the nuisance parameters, as they vary at each realization of the process.
}

two different $\mathrm{CRB}$ expressions can be derived: the conditional CRB (CCRB) and the unconditional CRB (UCRB). For the use and limitations of the CML and CCRB applied to timing recovery, see [10].

This paper focuses on the unconditional CRB (UCRB), as an alternative to the MCRB proposed in [1] for its use in synchronization problems. The main motivation of introducing the UCRB in timing recovery is that, in general, the MCRB cannot be attained, and more importantly, it is not adequate to account for the effect of low symbol transition densities on the data stream.

Using the resemblance of (2) with the model used in sensor array processing, the research in [8] was used in [9] to formulate the unconditional CRB (UCRB) for $\tau$ :

$$
\operatorname{UCRB}(\tau)=\frac{\sigma^{2}}{2 \operatorname{tr}(\mathbf{Y S})}
$$

where ${ }^{2}$

$$
\begin{aligned}
\mathbf{Y} & =\mathbf{D}_{\tau}^{T} \mathbf{P}_{\mathbf{A}_{\tau}}^{\perp} \mathbf{D}_{\tau} \\
\mathbf{S} & =\boldsymbol{\Gamma} \mathbf{A}_{\tau}^{T} \mathbf{R}_{\tau}^{-1} \mathbf{A}_{\tau} \boldsymbol{\Gamma} \\
\mathbf{R}_{\tau} & =\mathbf{A}_{\tau} \boldsymbol{\Gamma} \mathbf{A}_{\tau}^{T}+\sigma^{2} \mathbf{I} \\
\mathbf{P}_{\mathbf{A}_{\tau}}^{\perp} & =\mathbf{I}-\frac{T_{s}}{E_{g}} \mathbf{A}_{\tau} \mathbf{A}_{\tau}^{T} \\
\mathbf{D}_{\tau} & =\frac{d \mathbf{A}_{\tau}}{d \tau}
\end{aligned}
$$

where the $k$ th column of matrix $\mathbf{D}_{\tau}$ is formed by the samples of $g^{\prime}(t-k T-\tau)$, and $g^{\prime}(t)=d g(t) / d t$.

Of course, digital communications signals are non-Gaussian, as the data symbols take values from a finite alphabet of $N$ values, and then, the Gaussian UCRB is no longer a lower bound on the variance of consistent estimators, in the sense that it may differ from the true CRB in an unknown manner. The effect of the violation of this assumption has been explained in [10]. ${ }^{3}$ In short, the Gaussian assumption of the data symbols leads to a UCRB that is a valid lower bound on the variance of any consistent estimator that is quadratic with respect to the received signal, which is a property shared by most NDA timing recovery schemes derived in the literature. That is, the UCRB in (4) applies only to quadratic timing recovery schemes. Note, however, that at low $E_{s} / N_{o}$ conditions, the noise may predominate over the desired signal. In these conditions, the violation of the Gaussian assumption has a small effect on the validity of the Gaussian UCRB. In this respect, one of the results of this paper is showing that the Gaussian UCRB for timing estimation approaches asymptotically the true CRB at low $E_{s} / N_{o}$, irrespective of the nature of the estimator.

The main purpose of this contribution is the derivation of simple and meaningful expressions from (4) and (5). To this end, we first focus on the case of uncorrelated data symbols, which leads to an expression valid at any $E_{s} / N_{o}$. Second, we introduce the correlation of the data symbols and obtain asymptotic

\footnotetext{
${ }^{2}$ Note that in the case of infinite duration pulses, matrices $\mathbf{A}_{\tau}$ and $\mathbf{D}_{\tau}$ become semi-infinite. However, this does not modify the validity of the results presented through the paper.

${ }^{3}$ For an in-depth study of this topic, see [12, coroll. 1 and 2], and references therein.
} 
limits for low and high $E_{s} / N_{o}$. Finally, we develop expressions applicable at any $E_{s} / N_{o}$. The development will also offer an insightful connection between the work performed in [4] and [10].

\section{UCRB FOR UNCORRELATED DATA SYMBOLS}

Using property (48) (see Appendix A) in the case of uncorrelated data symbols $\left(\boldsymbol{\Gamma}=\sigma_{a}^{2} \mathbf{I}\right)$, and taking into account (3), we can write the UCRB from (4) as follows:

$$
\operatorname{UCRB}(\tau)=\frac{\sigma^{2}}{2 \operatorname{tr}(\mathbf{Y}) \frac{\boldsymbol{\sigma}_{a}^{4}}{\boldsymbol{\sigma}_{a}^{2}+\sigma^{2} \frac{T_{s}}{E_{g}}}} .
$$

Now, matrix $\mathbf{Y}$ can be written as

$$
\mathbf{Y}=\mathbf{D}_{\tau}^{T} \mathbf{P}_{\mathbf{A}_{\tau}}^{\perp} \mathbf{D}_{\tau}=\mathbf{D}_{\tau}^{T} \mathbf{D}_{\tau}-\frac{T_{s}}{E_{g}} \mathbf{B}_{\tau}^{T} \mathbf{B}_{\tau}
$$

where

$$
\mathbf{B}_{\tau}=\mathbf{A}_{\tau}^{T} \mathbf{D}_{\tau}
$$

Matrices $\mathbf{D}_{\tau}^{T} \mathbf{D}_{\tau}$ and $\mathbf{B}_{\tau}$ are $L \times L$ Toeplitz, and their diagonals have a common element that can be easily related with the following cross-correlation functions:

$$
\begin{aligned}
\overline{\operatorname{diag}}_{p}\left[\mathbf{D}_{\tau}^{T} \mathbf{D}_{\tau}\right] & =\frac{r_{g^{\prime}}(p T)}{T_{s}}=-\frac{r_{g}^{\prime \prime}(p T)}{T_{s}} \\
\overline{\operatorname{diag}}_{p}\left[\mathbf{B}_{\tau}\right] & =\frac{r_{g g^{\prime}}(p T)}{T_{s}}=\frac{r_{g}^{\prime}(p T)}{T_{s}}
\end{aligned}
$$

where $r_{g}(\tau)=\int g(t) g(t+\tau) d t, r_{g g^{\prime}}(\tau)=\int g(t) g^{\prime}(t+\tau) d t$, $r_{g^{\prime}}(\tau)=\int g^{\prime}(t) g^{\prime}(t+\tau) d t, r_{g}^{\prime}(\tau)=d r_{g}(\tau) / d \tau, r_{g}^{\prime \prime}(\tau)=$ $d^{2} r_{g}(\tau) / d \tau^{2}$, and $\overline{\operatorname{diag}}_{p}$ represents the common element of the $p$ th diagonal $(p=-(L-1), \ldots, 0, \ldots, L-1)$ of a Toeplitz matrix. From (9), the traces of $\mathbf{D}_{\tau}^{T} \mathbf{D}_{\tau}$ and $\mathbf{B}_{\tau}^{T} \mathbf{B}_{\tau}$ are

$$
\begin{aligned}
\operatorname{tr}\left[\mathbf{D}_{\tau}^{T} \mathbf{D}_{\tau}\right] & =L \overline{\operatorname{diag}}_{0}\left[\mathbf{D}_{\tau}^{T} \mathbf{D}_{\tau}\right]=\frac{-L r_{g}^{\prime \prime}(0)}{T_{s}} \\
\operatorname{tr}\left[\mathbf{B}_{\tau}^{T} \mathbf{B}_{\tau}\right] & =\operatorname{sum}\left(\mathbf{B}_{\tau} \odot \mathbf{B}_{\tau}\right) \\
& =\frac{1}{T_{s}^{2}} \sum_{m=-(L-1)}^{(L-1)}(L-|m|) r_{g}^{\prime 2}(m T)
\end{aligned}
$$

where $\odot$ denotes the Schur product (element-wise) between two matrices. ${ }^{4}$ Finally, we can write, from (7)

$$
\begin{aligned}
\operatorname{tr}(\mathbf{Y}) & =\frac{L S}{T_{s}} \\
S & =-r_{g}^{\prime \prime}(0)-\frac{1}{E_{g}} \sum_{m=-(L-1)}^{(L-1)} \frac{L-|m|}{L} r_{g}^{\prime 2}(m T) .
\end{aligned}
$$

${ }^{4}$ The property that $\operatorname{tr}\left(\mathbf{U}^{T} \mathbf{V}\right)=\operatorname{sum}(\mathbf{U} \odot \mathbf{V})$, where "sum" means the summation of all the elements of a matrix, has been used in (11).
Substituting in (6), we obtain

$$
\operatorname{UCRB}(\tau)=\frac{\sigma^{2} T_{s}\left(\sigma_{a}^{2}+\sigma^{2} \frac{T_{s}}{E_{g}}\right)}{2 L S \sigma_{a}^{4}}=\frac{N_{o}\left(1+\frac{1}{\frac{E_{s}}{N_{o}}}\right)}{L S \sigma_{a}^{2}}
$$

which after straightforward manipulation yields ${ }^{5}$

$$
\operatorname{UCRB}(\tau)=\frac{1}{8 \pi^{2} L \xi} T^{2} \frac{\frac{E_{s}}{N_{o}}+1}{\left(\frac{E_{s}}{N_{o}}\right)^{2}}
$$

where the factor $\xi$ is defined as

$$
\begin{aligned}
\xi & =\frac{T^{2}}{4 \pi^{2} E_{g}}\left(-r_{g}^{\prime \prime}(0)-\frac{1}{E_{g}} \sum_{m=-(L-1)}^{(L-1)} \frac{L-|m|}{L} r_{g}^{\prime 2}(m T)\right) \\
& \approx \frac{T^{2}}{4 \pi^{2} E_{g}}\left(-r_{g}^{\prime \prime}(0)-\frac{1}{E_{g}} \sum_{m=-\infty}^{\infty} r_{g}^{\prime 2}(m T)\right) .
\end{aligned}
$$

The last term of (14) represents an approximation for large $L\left(L \gg N_{p}\right)$, where $N_{p}$ is the effective one-sided length of the sequence $r_{g}(m T)$, that is, $\left|r_{g}(\tau) / r_{g}(0)\right| \approx 0$ for $|\tau|>N_{p} T$. Note that for low $E_{s} / N_{o}$, we obtain

$$
\begin{aligned}
\operatorname{UCRB}(\tau)_{\text {low }} & \approx \frac{1}{2 L} \frac{1}{\left(\frac{E_{s}}{N_{o}}\right)^{2}} \\
& \times\left(-\frac{1}{E_{g}} r_{g}^{\prime \prime}(0)-\frac{1}{E_{g}^{2}} \sum_{m=-\infty}^{\infty} r_{g}^{\prime 2}(m T)\right)^{-1}
\end{aligned}
$$

in agreement with the expression derived in [4], 6 thus confirming that the $\operatorname{UCRB}(\tau)_{\text {low }}$ becomes asymptotically the true CRB for low $E_{s} / N_{o}{ }^{7}$ Equation (13) becomes then a generalization of [4] in the sense that it is valid for all $E_{s} / N_{o}$. However, we observe from (13) that when the $E_{s} / N_{o}$ is above the threshold of $\left(E_{s} / N_{o}\right)_{\mathrm{th}}=0 \mathrm{~dB}$, the performance limit tends to be inversely proportional to $E_{s} / N_{o}$, although, as we have already explained, this is only applicable to quadratic timing recovery schemes. Therefore, we have found that the pulse-dependent factor $\xi$ in (14) obtained also in [4] is not only relevant at low $E_{s} / N_{o}$, but it also plays an important role in the performance that can be attained by quadratic timing recovery schemes at any $E_{s} / N_{o}$.

${ }^{5}$ The manipulation of (12) to obtain (13) is done with the intention of obtaining an expression with a similar notation employed by D'Andrea et al. in $[1,(32)]$, taking into account that $L$ can be easily related with the equivalent noise bandwidth $B_{L}$ of a closed-loop scheme as $L=1 /\left(2 B_{L} T\right)$. The same applies to (21), (22) and (25), (26) in the next section.

${ }^{6}$ To make this comparison, see the second term of [4, eq. (13)]. It should be noted, however, that the CRB defined in [4] represents a lower bound on the normalized timing error (normalized by the symbol interval $T$ ), which explains the presence of the factor $T^{-2}$ in that equation. It is finally noted that the expression in [4] is implicitly assuming that $E_{g}=1$.

${ }^{7}$ It is noted that in [4], the authors considered the low-SNR limit of the joint likelihood function of timing, phase, and data symbols, averaged over the true (non-Gaussian) statistics of the data symbols and a uniformly distributed (in $[-\pi, \pi))$ carrier phase. For low-SNR, this averaged likelihood function becomes quadratic in the received signal. This result is coherent with the fact that the UCRB (only valid for quadratic estimators in the general case) becomes the true CRB at low SNR. 
As remarked in [4], the tracking error variance at low $E_{s} / N_{o}$ of the popular NDA noncarrier-aided filter and square timing recovery algorithm $[13 \text {, Sec. 6.3.6., pag. 356, Eq. 6-150) }]^{8}$ (see the noise-by-noise term in [13, p. 357, Eq. 6-166]) equals $\operatorname{UCRB}(\tau)_{\text {low }}$ in (15), which indicates that this algorithm is optimum at small $E_{s} / N_{o}$. Now, the study performed in the present contribution allows us to make an additional remark for this algorithm. Note that for high $E_{s} / N_{o}$, we obtain

$$
\operatorname{UCRB}(\tau)_{\text {high }} \approx \frac{1}{2 L} \frac{1}{\frac{E_{s}}{N_{o}}}\left(-\frac{1}{E_{g}} r_{g}^{\prime \prime}(0)-\frac{1}{E_{g}^{2}} \sum_{m=-\infty}^{\infty} r_{g}^{\prime 2}(m T)\right)^{-1}
$$

which is identical to the signal-by-noise term of $[13$, p. 358 , Eq. 6-170]. This suggests that this algorithm would be the optimal quadratic algorithm if the self-noise (signal-by-signal term) were not present. As is well known [13, p. 354 Eq. 6-140], the self-noise of the square timing recovery algorithm is proportional to the square of the normalized synchronizer bandwidth $B_{L} T=1 /(2 L)$. Given this fact, we can conclude that this algorithm is asymptotically (for sufficiently large $L$ ) the best quadratic NDA synchronizer because the self-noise becomes insignificant with respect to the other terms as $L$ increases.

Now, we establish the connection of this analysis with the work carried out in [10]. The quadratic conditional ML (CML) timing recovery scheme developed in [10] is shown to attain the $\operatorname{UCRB}(\tau)_{\text {high }}$ at high $E_{s} / N_{o}$ and to be self-noise free. ${ }^{9}$ However, the CML scheme may be not optimum at low $E_{s} / N_{o}$, thus becoming the counterpart of the square timing recovery algorithm, which does in fact attain the $\operatorname{UCRB}(\tau)_{\text {low }}$ at low $E_{s} / N_{o}$, but it may be affected by self noise at high $E_{s} / N_{o}$. We also note that [10] proves that, in the case of square-root raised cosine pulses with roll-off factor $\alpha>0$ (see Fig. 1 for uncorrelated data $\gamma=0)$, the value of $\xi$ tends to $\alpha / 8$ for large $L\left(L \gg N_{p}\right)$ and that $\operatorname{UCRB}(\tau)_{\text {high }}$ tends to the conditional CRB $(C C R B(\tau))$ as the $E_{s} / N_{o}$ increases.

Finally, from (16), we can write the following inequality:

$$
\begin{aligned}
\operatorname{UCRB}(\tau)_{h i g h} & >\frac{1}{2 L} \frac{1}{\frac{E_{s}}{N_{o}}}\left(-\frac{1}{E_{g}} r_{g}^{\prime \prime}(0)\right)^{-1} \\
& =M C R B(\tau) \\
& =\frac{1}{8 \pi^{2} L \xi^{\prime}} \frac{T^{2}}{\frac{E_{s}}{N_{o}}}
\end{aligned}
$$

where $\xi^{\prime}=(1 / 4)\left((1 / 3)+\alpha^{2}\left(1-\left(8 / \pi^{2}\right)\right)\right.$, thus showing the connection with the MCRB [1], [14].

\section{ASYMPTOTIC UCRBS FOR CORRELATED DATA SYMBOLS}

\section{A. Low-SNR Approximation}

At low SNR, we can make the approximation $\mathbf{R}_{\tau}^{-1}=$ $\left(\mathbf{A}_{\tau} \boldsymbol{\Gamma} \mathbf{A}_{\tau}^{T}+\sigma^{2} \mathbf{I}\right)^{-1} \approx \sigma^{-2} \mathbf{I}$ in (5), leading from (4) to

$$
\operatorname{UCRB}(\tau)_{\text {low }}=\frac{\sigma^{4} T_{s}}{2 E_{g} \operatorname{sum}\left(\mathbf{Y} \odot \Gamma^{2}\right)}
$$

${ }^{8}$ It is noted that this algorithm appears in different papers and text books with different names. To clarify this, we also cite [14, Sec. 8.3.1., pag. 429, Eq. 8.3.5.], where it is called ML-Oriented-NDA TED, and [9] and [10], where it is called low-SNR-UML TED.

${ }^{9}$ See also [15], where the modified ML and minimum mean-square error approaches are proposed to obtain self-noise free synchronizers.

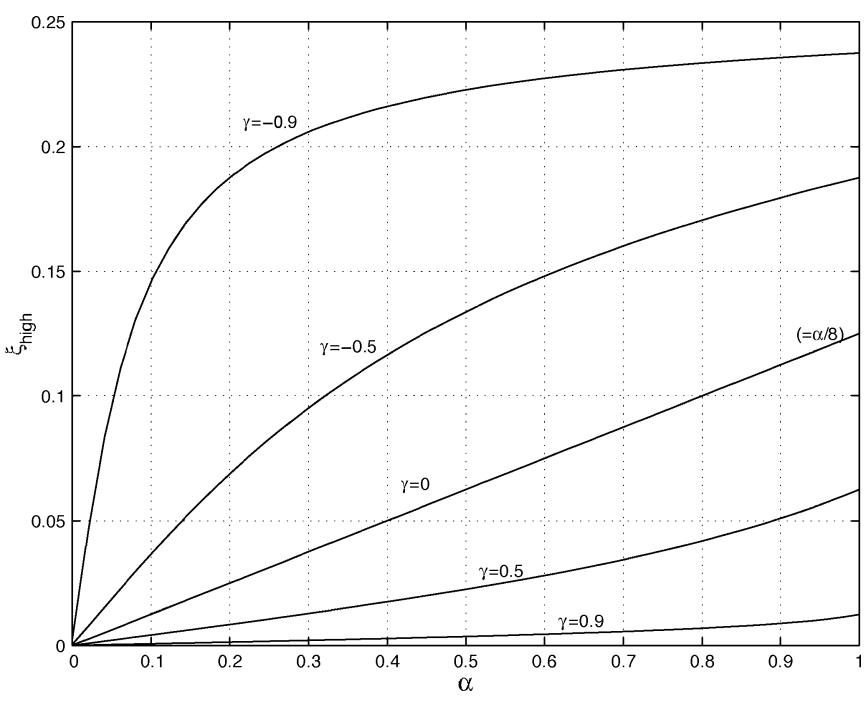

Fig. 1. $\xi_{\text {high }}$ as a function of the roll-off parameter $\alpha$ for different values of the symbol correlation factor $\gamma \cdot \gamma=0$ represents the case of uncorrelated data.

where (3) has also been used. For large $L\left(L \gg N_{a}\right)$, where $N_{a}$ is the effective one-sided length of the sequence $r_{a}[\mathrm{~m}]$, that is, $\left|r_{a}[m] / r_{a}[0]\right| \approx 0$ for $m>N_{a}$, we can write the following approximation for the diagonals of a matrix product:

$$
\begin{aligned}
& \overline{\operatorname{diag}}_{p}\left[\boldsymbol{\Gamma}^{2}\right] \approx q_{a}[p] \\
& \quad=\sum_{m=-\infty}^{\infty} r_{a}[m] r_{a}^{*}[m+p] \\
& T_{s}^{2} \overline{\operatorname{diag}}_{p}\left[\mathbf{B}_{\tau}^{T} \mathbf{B}_{\tau}\right] \approx q_{g}[p] \\
& \quad=\sum_{m=-\infty}^{\infty} r_{g}^{\prime}(m T) r_{g}^{\prime}((m+p) T)
\end{aligned}
$$

where $\mathbf{B}_{\tau}$ is given in (8). Then, using (9), (19), and (20), we can write

$$
\begin{aligned}
\operatorname{sum}\left(\mathbf{Y} \odot \boldsymbol{\Gamma}^{2}\right) & \approx \frac{L S_{\text {low }}}{T_{s}} \\
S_{\text {low }} & =\sum_{p=-\infty}^{\infty}\left(-r_{g}^{\prime \prime}(p T)-\frac{1}{E_{g}} q_{g}[p]\right) q_{a}[p] .
\end{aligned}
$$

Substituting in (18), we obtain

$$
\operatorname{UCRB}(\tau)_{\text {low }}=\frac{\sigma^{4} T_{s}^{2}}{2 L E_{g} S_{\text {low }}}=\frac{2 N_{o}^{2}}{L E_{g} S_{\text {low }}}
$$

which after straightforward manipulation yields

$$
\operatorname{UCRB}(\tau)_{\text {low }}=\frac{1}{8 \pi^{2} L \xi_{\text {low }}} \frac{T^{2}}{\left(\frac{E_{s}}{N_{o}}\right)^{2}}
$$

where the factor $\xi_{\text {low }}$ is defined as

$$
\xi_{\text {low }}=\frac{T^{2}}{4 \pi^{2} E_{g}} \sum_{p=-\infty}^{\infty}\left(-r_{g}^{\prime \prime}(p T)-\frac{1}{E_{g}} q_{g}[p]\right) \frac{q_{a}[p]}{\sigma_{a}^{4} .}
$$

Note that obtained (22) and (23), constitute a generalization of [4] for the case of correlated data. 


\section{B. High SNR Approximation}

At high SNR, we can make the approximation $\mathbf{S}=$ $\boldsymbol{\Gamma} \mathbf{A}_{\tau}^{T}\left(\mathbf{A}_{\tau} \boldsymbol{\Gamma} \mathbf{A}_{\tau}^{T}+\sigma^{2} \mathbf{I}\right)^{-1} \mathbf{A}_{\tau} \boldsymbol{\Gamma} \approx \boldsymbol{\Gamma}$ (see Appendix A) ${ }^{10}$ in (5), leading from (4) to

$$
\operatorname{UCRB}(\tau)_{\text {high }}=\frac{\sigma^{2}}{2 \operatorname{sum}(\mathbf{Y} \odot \Gamma)}
$$

Following the same procedure as in the previous subsection, we can write

$$
\begin{aligned}
\operatorname{sum}(\mathbf{Y} \odot \boldsymbol{\Gamma}) & \approx \frac{L S_{\text {high }}}{T_{s}} \\
S_{\text {high }} & =\sum_{p=-\infty}^{\infty}\left(-r_{g}^{\prime \prime}(p T)-\frac{1}{E_{g}} q_{g}[p]\right) r_{a}[p] .
\end{aligned}
$$

Substituting in (24), we obtain

$$
\operatorname{UCRB}(\tau)_{\text {high }}=\frac{\sigma^{2} T_{s}}{2 L S_{\text {high }}}=\frac{N_{o}}{L S_{\text {high }}}
$$

which after straightforward manipulation yields

$$
\operatorname{UCRB}(\tau)_{\text {high }}=\frac{1}{8 \pi^{2} L \xi_{\text {high }}} \frac{T^{2}}{\frac{E_{s}}{N_{o}}}
$$

where the factor $\xi_{\text {high }}$ is defined as

$$
\xi_{\text {high }}=\frac{T^{2}}{4 \pi^{2} E_{g}} \sum_{p=-\infty}^{\infty}\left(-r_{g}^{\prime \prime}(p T)-\frac{1}{E_{g}} q_{g}[p]\right) \frac{r_{a}[p]}{\sigma_{a}^{2}} .
$$

The evolution of factor $\xi_{\text {high }}$ as a function of the roll-off $\alpha$ and the correlation factor $\gamma \in[-1,1]$ [defined in the next section in (30)] is shown in Figs. $1^{11}$ and 2, respectively. It is shown in both figures that $\xi_{\text {high }}$ increases with $\alpha$ (the excess-bandwidth) and decreases with the correlation factor $\gamma$, that is, for low transition densities. It is also noted from Fig. 2 that $\xi_{\text {high }}=0.25$ for $\gamma=-1$ at any $\alpha$ and that $\xi_{\text {high }}=(1-\gamma) / 8$ for $\alpha=1$.

\section{Threshold Characterization}

The threshold $E_{s} / N_{o}[9]\left(E_{s} / N_{o}\right)_{\text {th }}$ is defined as the $E_{s} / N_{o}$ below which the performance starts to be inversely proportional to $\left(E_{s} / N_{o}\right)^{2}$. This threshold can be easily found by solving for the equation $\operatorname{UCRB}(\tau)_{\text {high }}=\operatorname{UCRB}(\tau)_{\text {low }}$, which, using (22) and (26), yields

$$
\left(\frac{E_{s}}{N_{o}}\right)_{\text {th }}=\frac{\xi_{\text {high }}}{\xi_{\text {low }}}
$$

The determination of this threshold is meaningful, as it gives a minimum value for the $E_{s} / N_{o}$ below which the estimation of the corresponding parameter becomes inherently a difficult task. The practical importance in the case of timing estimation is that

\footnotetext{
${ }^{10}$ This approximation is obtained simply by making $\sigma^{2}=0$ in (48).

${ }^{11}$ It is noted that this result generalizes the result of $\xi_{\text {high }}=\alpha / 8$ for uncor-
} related data $(\gamma=0)$ derived in $[10,(32)]$.

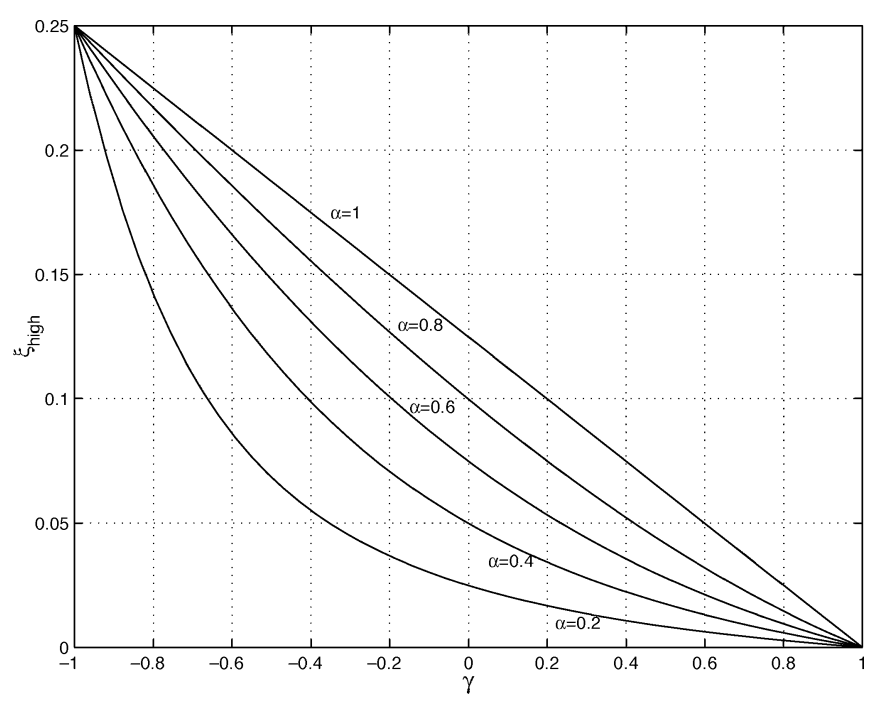

Fig. 2. $\xi_{\text {high }}$ as a function of the symbol correlation factor $\gamma$ for different values of the roll-off parameter $\alpha$.

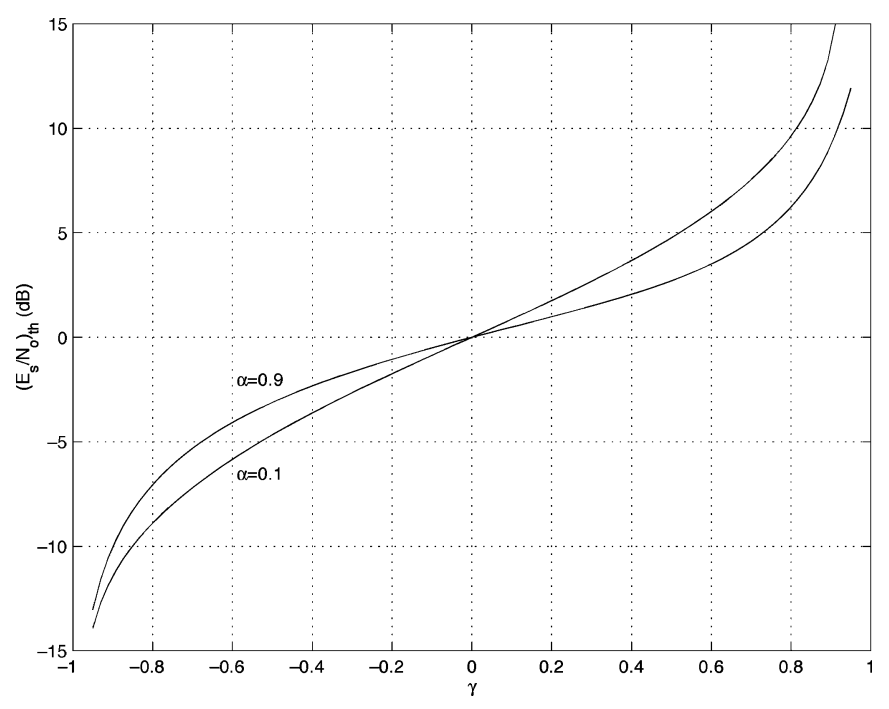

Fig. 3. Evolution of the threshold $E_{s} / N_{o},\left(E_{s} / N_{o}\right)$ th as a function of the correlation factor $\gamma$ for $\alpha=0.1$ and $\alpha=0.9$.

if the operating $E_{s} / N_{o}$ is smaller than $\left(E_{s} / N_{o}\right)_{\text {th }}$, a reduction of $3 \mathrm{~dB}$ in the operating $E_{s} / N_{o}$ cannot be compensated for, simply by duplicating the observation interval, revealing a high noise sensitivity in this operating range. Therefore, a threshold reduction caused by a change of a certain signal parameter will indicate an increased difficulty for the timing estimation problem under these conditions. The determination of $\left(E_{s} / N_{o}\right)_{\text {th }}$ also establishes quantitatively the meaning of low-SNR and high-SNR for a given scenario, thus giving the range of validity of some low-SNR limits of the CRB derived in the literature [4].

The evolution of $\left(E_{s} / N_{o}\right)$ th as a function of $\gamma$ is depicted in Fig. 3. It is seen that although $\left(E_{s} / N_{o}\right)_{\text {th }}=0 \mathrm{~dB}$ for uncorrelated data, it increases for positive correlation factors (low transition densities), and it decreases for negative correlation factors (high transition densities). The impact of $\gamma$ on the threshold is more significant for small roll-off factors. 


\section{TRANSITION DENSITY MODEL}

This section develops a simple model for the autocorrelation of the data symbols $r_{a}[p]$ to account for the transition density of the actual symbol stream. A Markov chain is adopted for that purpose. Specifically, the conditional probability that the symbol at the instant $(k+1) T$ be $a^{(q)}$ when the symbol at the instant $k T$ was $a^{(n)}$ is characterized as follows:

$$
P\left(\left(a_{k+1}=a^{(q)}\right) /\left(a_{k}=a^{(n)}\right)\right)= \begin{cases}1-\beta, & n=q \\ \frac{\beta}{(N-1)}, & n \neq q\end{cases}
$$

where $n, q \in[0, N-1]$, and $\beta$ is the probability of a symbol transition or transition density. As the assumed transition probabilities to the remaining $(n \neq q)$ symbols are equal, the generated process is stationary, and the symbols are equiprobable. ${ }^{12}$ It is well-known [16] that the correlation of the symbols can be computed as

$$
\begin{aligned}
r_{a}[p] & =E\left\{a_{k} a_{k+p}^{*}\right\}=\sigma_{a}^{2} \gamma^{|p|} \\
\gamma & =\frac{r_{a}[1]}{\sigma_{a}^{2}} .
\end{aligned}
$$

It is not difficult to show that $r_{a}[p]$ has an approximate time support of $N_{a}=(1 / 2(1-|\gamma|))$. Decomposing the expectation in (30), we can write

$$
\begin{aligned}
\gamma & =\frac{1}{\sigma_{a}^{2} N} \sum_{m=0}^{N-1} a^{(m)} E\left\{a_{k+1} \mid a_{k}=a^{(m)}\right\} \\
& =\frac{1}{\sigma_{a}^{2} N} \sum_{m=0}^{N-1} a^{(m)}\left((1-\beta) a^{(m)}+\frac{\beta}{N-1} \sum_{n \neq m} a^{(n)}\right) \\
& =\frac{1}{\sigma_{a}^{2} N} \sum_{m=0}^{N-1} a^{(m)}\left((1-\beta) a^{(m)}-\frac{\beta}{N-1} a^{(m)}\right) \\
& =\frac{1}{\sigma_{a}^{2} N} \sum_{m=0}^{N-1}\left(a^{(m)}\right)^{2}\left(1-\frac{\beta N}{N-1}\right) \\
& =\left(1-\frac{\beta N}{N-1}\right)
\end{aligned}
$$

To better understand the physical meaning of $\beta$ looking at (31), let us focus on the following particular examples.

- $\beta=0 \rightarrow \gamma=1 \rightarrow r_{a}[p]=\sigma_{a}^{2}$. The symbol sequence is constant. No transitions are present.

- $\beta=(1-1 / N) \rightarrow \gamma=0 \rightarrow r_{a}[p]=\sigma_{a}^{2} \delta_{p}$. The symbol sequence is uncorrelated. Note that for $N=2 \rightarrow$ $\beta=1 / 2$, which means that for binary random digital modulation, the symbol transition density is $50 \%$.

- $\left.\begin{array}{c}\beta=1 \\ N=2\end{array}\right\} \rightarrow \gamma=-1 \rightarrow r_{a}[p]=\sigma_{a}^{2}(-1)^{p}$. The symbol sequence is the alternated pattern $\left\{a_{k}\right\}=1,-1,1,-1, \ldots$, or $\left\{a_{k}\right\}=-1,1,-1,1, \ldots$ The symbol transition density is $100 \%$.

\footnotetext{
${ }^{12}$ Other models can be found in the literature that account for the symbol transition density. For instance, in [13, pp. 187-189], a model with independent but nonequiprobable binary data symbols was formulated for synchronizer performance evaluation. In contrast, the model proposed in (29) assumes equiprobable but nonindependent data symbols. The proposed model (29) is more general in two senses: i) It is also adequate to model transition densities above $50 \%$, and ii) it is adequate for any alphabet size $(N)$ (not only binary data).
}

Finally, it is not difficult to show that the function $q_{a}[p]$ defined in (19) becomes

$$
q_{a}[p] \approx \begin{cases}\sigma_{a}^{4} \gamma^{|p|}\left(|p|-1+\frac{2}{1-\gamma^{2}}\right), & \text { for } L \gg N_{a} \\ \sigma_{a}^{4} L \gamma^{|p|}, & \text { for } L \ll N_{a}\end{cases}
$$

\section{GENERALIZATION OF THE UCRB}

In this section, we finally give a procedure for the evaluation of the $\operatorname{UCRB}(\tau)$ at any $E_{s} / N_{o}$ and at any degree of symbol autocorrelation. It is shown in Appendix A that matrix $\mathbf{S}$ in (4) can be expressed as $\mathbf{S}=\Gamma \boldsymbol{\Omega}^{-1} \boldsymbol{\Gamma}$, where $\boldsymbol{\Omega}=\boldsymbol{\Gamma}+\sigma^{2}\left(\mathbf{A}_{\tau}^{T} \mathbf{A}_{\tau}\right)^{-1}$. Then, the term $\operatorname{tr}(\mathbf{Y S})$ in (4) for large $L\left(L \gg N_{a}\right)$ can be expressed as ${ }^{13}$

$$
\begin{aligned}
\operatorname{tr}(\mathbf{Y S}) & =\operatorname{tr}\left(\mathbf{Y} \boldsymbol{\Gamma} \mathbf{\Omega}^{-1} \boldsymbol{\Gamma}\right) \\
& =L \int_{\frac{-1}{2}}^{\frac{1}{2}} Y(F) R_{a}(F) \Omega^{-1}(F) R_{a}(F) d F
\end{aligned}
$$

where

$$
\begin{aligned}
Y(F) & =\sum_{p=-\infty}^{\infty} y[p] e^{-j 2 \pi F p} \\
R_{a}(F) & =\sum_{p=-\infty}^{\infty} r_{a}[p] e^{-j 2 \pi F p} \\
\Omega(F) & =R_{a}(F)+\frac{\sigma^{2} T_{s}}{E_{g}}
\end{aligned}
$$

where $y[p]$ and $r_{a}[p]$ are the common element of the $p$ th diagonal of Toeplitz matrices $\mathbf{Y}$ and $\Gamma$, respectively. More compactly, we can write

$$
\operatorname{tr}(\mathbf{Y S})=L \int_{\frac{-1}{2}}^{\frac{1}{2}} Y(F) S_{a}(F) d F
$$

where

$$
S_{a}(F)=\frac{\left|R_{a}(F)\right|^{2}}{R_{a}(F)+\frac{\sigma^{2} T_{s}}{E_{g}}}=\sigma_{a}^{2} \frac{\left|\bar{R}_{a}(F)\right|^{2}}{R_{a}(F)+\left(\frac{E_{s}}{N_{o}}\right)^{-1}}
$$

where $\bar{R}_{a}(F)$ is the normalized discrete Fourier transform of $r_{a}[p]$, which is defined as $\bar{R}_{a}(F)=\left(1 / \sigma_{a}^{2}\right) R_{a}(F)$. Using the transition density model developed in the previous section, it is not difficult to show that $\bar{R}_{a}(F)$ can be expressed as

$$
\bar{R}_{a}(F)=\frac{1}{\sigma_{a}^{2}} \frac{1-\gamma^{2}}{\left|1-\gamma e^{-j 2 \pi F}\right|^{2}} .
$$

Now, defining $s_{a}[p]=\int_{-1 / 2}^{1 / 2} S_{a}(F) e^{j 2 \pi F p} d F$, which can be numerically computed in practice by means of the IFFT algorithm, we can use the Parseval's theorem to write $\operatorname{tr}(\mathbf{Y S})=$ $L \sum_{p=-\infty}^{\infty} y[p] s_{a}[p]$. Taking into account the expression of

${ }^{13}$ See [10, App. B, Prop. B.3] for a detailed proof of (33). 
matrix $\mathbf{Y}$ in (7), we can use (9) and (20) to write the coefficients $y[p]$ (the diagonals of $\mathbf{Y}$ ) as

$$
y[p]=\frac{1}{T_{s}}\left(-r_{g}^{\prime \prime}(p T)-\frac{1}{E_{g}} q_{g}[p]\right) .
$$

Substituting (38) in (4), we can write

$$
\begin{aligned}
\operatorname{UCRB}(\tau) & =\frac{\sigma^{2} T_{s}}{2 L S} \\
& =\frac{N_{o}}{L S} \\
S & =\sum_{p=-\infty}^{\infty}\left(-r_{g}^{\prime \prime}(p T)-\frac{1}{E_{g}} q_{g}[p]\right) s_{a}[p] .
\end{aligned}
$$

Finally, straightforward manipulation of (39) yields

$$
\operatorname{UCRB}(\tau)=\frac{1}{8 \pi^{2} L \xi} T^{2} \frac{\frac{E_{s}}{N_{o}}+1}{\left(\frac{E_{s}}{N_{o}}\right)^{2}}
$$

where

$$
\xi=\frac{T^{2}}{4 \pi^{2} E_{g}} \frac{\frac{E_{s}}{N_{o}}+1}{\frac{E_{s}}{N_{o}}} \sum_{p=-\infty}^{\infty}\left(-r_{g}^{\prime \prime}(p T)-\frac{1}{E_{g}} q_{g}[p]\right) \frac{s_{a}[p]}{\sigma_{a}^{2}} .
$$

In this manner, thanks to the inclusion of the term $\left(E_{s} / N_{o}+1\right)$ in both (40) and (41), we get in (40) an expression similar to (13), but now, it is valid for correlated symbols and for any range of $E_{s} / N_{o}$. The only difference between (40) and (13) is that the factor $\xi$ in (40) is now generalized in (41) for correlated data with respect to that derived in (14). Note that for the particular case of uncorrelated data, the coefficients $s_{a}[p]$ (the inverse Fourier transform of $S_{a}(f)$ in (36) for $\bar{R}_{a}(F)=1$ ) in (41) reduce to

$$
s_{a}[p]=\sigma_{a}^{2} \frac{\frac{E_{s}}{N_{o}}}{\frac{E_{s}}{N_{o}}+1} \delta_{p}
$$

and then, (41) reduces to (14). Note also that the obtained (40) and (41) constitute a generalization of previous work in [9] and [4] for the case of correlated data.

Figs. 4 and 5 show the evolution of the $\operatorname{UCRB}(\tau)$ normalized to the symbol interval as a function of the $E_{s} / N_{o}$, along with the high and low SNR approximation and the threshold points for different values of $\gamma$. It is seen that data streams with low transition densities $(\gamma>0)$ lead to a performance degradation that is more significant for small roll-off values. It is also observed that for $\gamma=-1$, the roll-off factor $\alpha$ has no influence on the bound.

\section{UCRB FOR FUlly CORRELATED DATA SyMBOL StREAMS}

Finally, this section addresses the particular case of fully correlated data streams. Examples are the constant and alternated symbol patterns for which $|\gamma|=1$. In these cases, matrix $\Gamma$ degenerates to rank-one, and it can be written as

$$
\boldsymbol{\Gamma}=\sigma_{a}^{2} \mathbf{u u}^{T}
$$

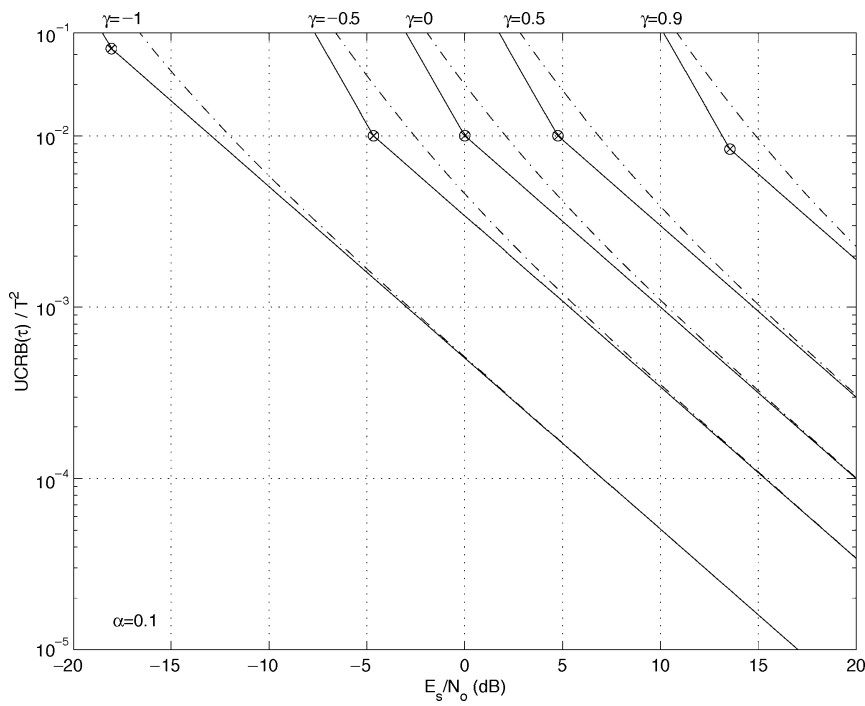

Fig. 4. Normalized $\operatorname{UCRB}(\tau)$ (dashed lines) as a function of the $E_{s} / N_{o}$ for different values of $\gamma . L=64 . \alpha=0.1$. The normalized $\operatorname{UCRB}(\tau)_{\text {low }}$ and $\operatorname{UCRB}(\tau)_{\text {high }}$ are also depicted (solid lines), along with the $\left(E_{s} / N_{o}\right)_{\text {th }}$ (cross point).

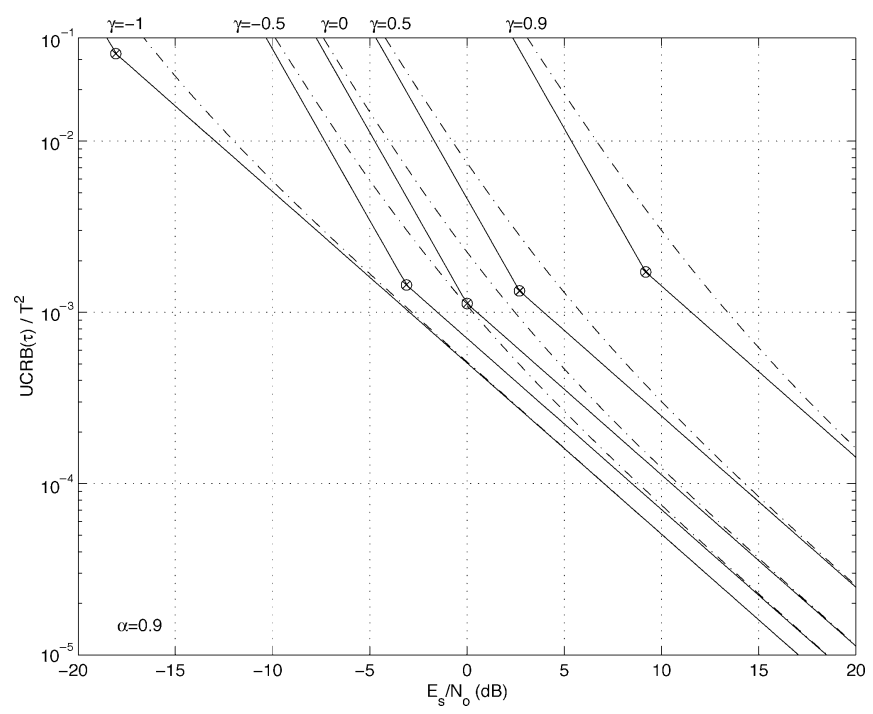

Fig. 5. Normalized $\operatorname{UCRB}(\tau)$ (dashed lines) as a function of the $E_{s} / N_{o}$ for different values of $\gamma . L=64 . \alpha=0.9$. The normalized $\operatorname{UCRB}(\tau)_{\text {low }}$ and $\operatorname{UCRB}(\tau)_{\text {high }}$ are also depicted (solid lines), along with the $\left(E_{s} / N_{o}\right)_{\text {th }}$ (cross point).

where $\mathbf{u}=[1,1,1,1 \ldots]^{T}$ for $\gamma=1$, or $\mathbf{u}=[1,-1,1,-1 \ldots]^{T}$ for $\gamma=-1$. In that case (see Appendix B), we can write

$$
\mathbf{S}=\boldsymbol{\Gamma}\left(\boldsymbol{\Gamma}+\sigma^{2}\left(\mathbf{A}_{\tau}^{T} \mathbf{A}_{\tau}\right)^{-1}\right)^{-1} \Gamma=\Gamma \frac{\frac{L E_{s}}{N_{o}}}{1+\frac{L E_{s}}{N_{o}}} .
$$

This means that for $|\gamma|=1$, the coefficients of $s_{a}[p]$ (the asymptotic diagonals of $\mathbf{S}$ ) in (41) are

$$
s_{a}[p]=\sigma_{a}^{2} \gamma^{|p|} \frac{\frac{L E_{s}}{N_{o}}}{1+\frac{L E_{s}}{N_{o}}} .
$$




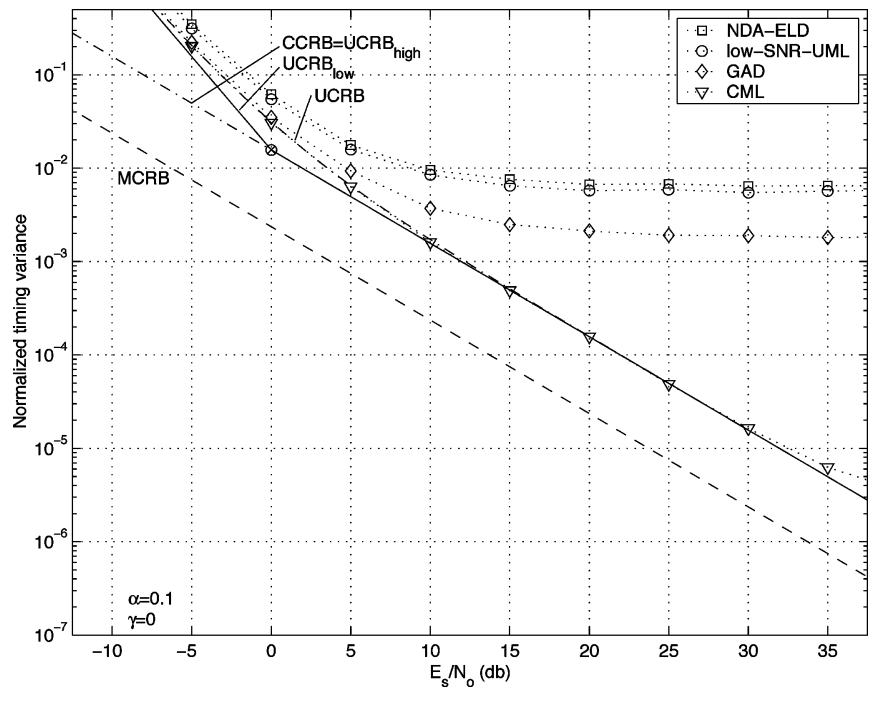

Fig. 6. Comparison of the performance of some quadratic timing recovery schemes with the new bounds for $L=64, \alpha=0.1$, and $\gamma=0$.

Using (41) and (45) in (40), we obtain

$$
\operatorname{UCRB}(\tau)_{\text {full }}=\frac{1}{8 \pi^{2} L \xi_{\text {full }}} \frac{T^{2}}{\frac{E_{s}}{N_{o}}} \frac{1+\frac{L E_{s}}{N_{o}}}{\frac{L E_{s}}{N_{o}}}
$$

where

$$
\xi_{\text {full }}=\frac{T^{2}}{4 \pi^{2} E_{g}} \sum_{p=-\infty}^{\infty}\left(-r_{g}^{\prime \prime}(p T)-\frac{1}{E_{g}} q_{g}[p]\right) \gamma^{|p|} .
$$

An important remark for the case of fully correlated data streams is that the threshold $E_{s} / N_{o}$ below which the performance starts to be inversely proportional to $\left(E_{s} / N_{o}\right)^{2}$ is $\left(E_{s} / N_{o}\right)_{\text {th }}=1 / L$, irrespective of the roll-off value, as it can be easily deduced from the mathematical relation obtained in (46). For instance, it can be seen in both Figs. 4 and 5 that the threshold (cross point) for $\gamma=-1$ and $L=64$ is $10 \log (1 / 64) \approx-18 \mathrm{~dB}$. The threshold, then, decreases as $L$ increases, and it becomes smaller than $0 \mathrm{~dB}$, which would be the threshold value in the presence of uncorrelated data, as also seen in these figures. This threshold-reduction effect can be helpful in the design of preamble lengths of alternated data intended solely for timing acquisition in very low $E_{s} / N_{o}$ scenarios. A final remark is that, in the case of $\gamma=1$ (the constant symbol pattern), $\xi_{\text {full }}=0$ for square-root raised cosine pulses, the physical meaning being that the modulated signal becomes constant and, therefore, insensitive to timing offsets.

\section{COMPARISON WITH QUADRATIC TIMING RECOVERY SCHEMES}

The derived performance bounds are here compared with the actual performance of some well-known quadratic timing recovery feedback schemes in the presence of uncorrelated and correlated data. Four timing error detectors are considered: the non-data-aided early-late detector (NDA-ELD) (see [14, Sec. 8.3.1., p. 429, Eq. 8.3.7.]), the low-SNR-UML (or ML-oriented) detector (see [9] and [10]), the Gardner detector (GAD) (see [14, Sec. 8.3.2., p. 431, Eq. 8.3.19]), and the CML detector (see [10]). Figs. 6-11 show the normalized timing variance of all

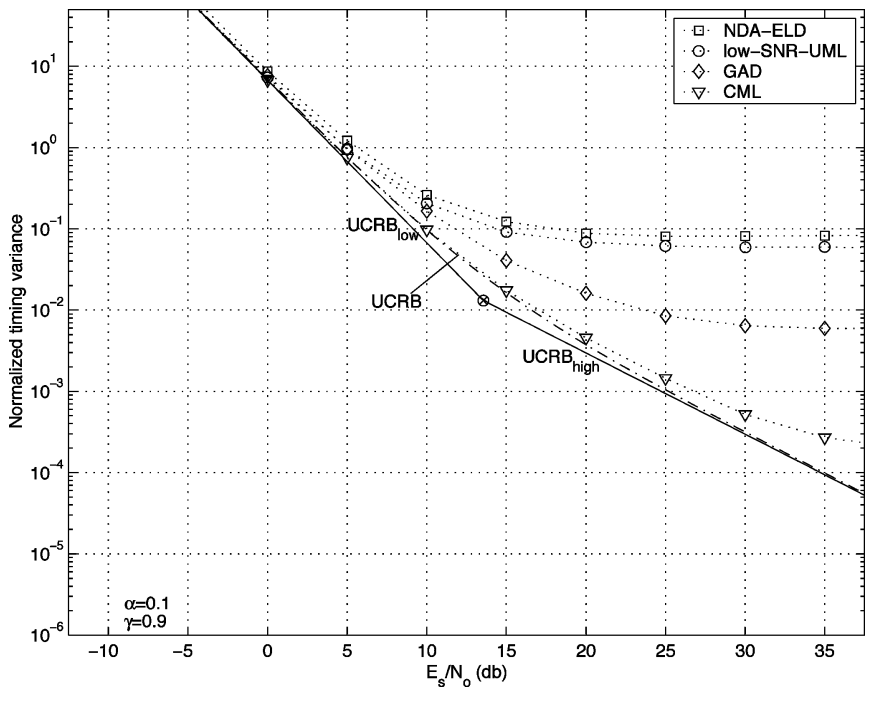

Fig. 7. Comparison of the performance of some quadratic timing recovery schemes with the new bounds for $L=64, \alpha=0.1$, and $\gamma=0.9$.

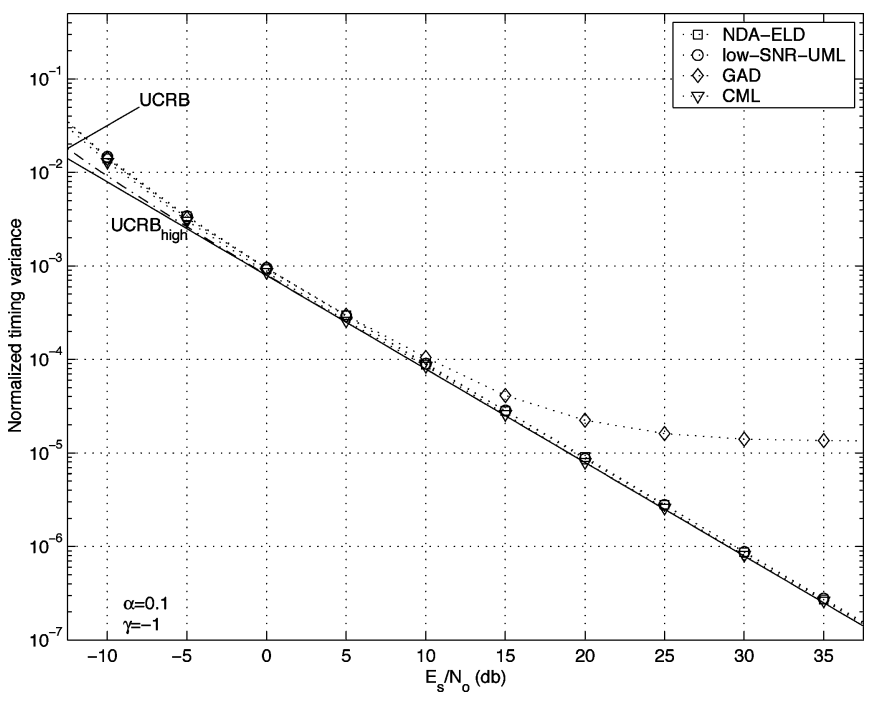

Fig. 8. Comparison of the performance of some quadratic timing recovery schemes with the new bounds, for $L=64, \alpha=0.1$, and $\gamma=-1$.

timing error detectors against $E_{s} / N_{o}$ using 5000 iterations with $L=64$, along with the UCRB and their asymptotic limits.

Fig. 6 corresponds to the case of uncorrelated data. It is seen that only the CML detector attains the UCRB (for moderate $E_{s} / N_{o}$ ). The remaining detectors exhibit a very high floor effect for this small roll-off factor. The MCRB is also depicted, which departs significantly from the UCRB. Fig. 7 corresponds to the case of a low transition density. The simulations confirm the existence of an increased threshold effect for that case, approaching all the estimators to the $\mathrm{UCRB}_{\text {low }}$ asymptote below this threshold. The case of $100 \%$ transition density is shown in Fig. 8, where, apart from the GAD, the detectors attain the UCRB for moderate and high $E_{s} / N_{o}$, and they exhibit a reduced threshold effect. However, all them tend to depart slightly from the UCRB at very low $E_{s} / N_{o}$.

Figs. 9-11 are similar to Figs. 6-8, respectively, but for the case of a high roll-off factor. The floor effect of all estimators 


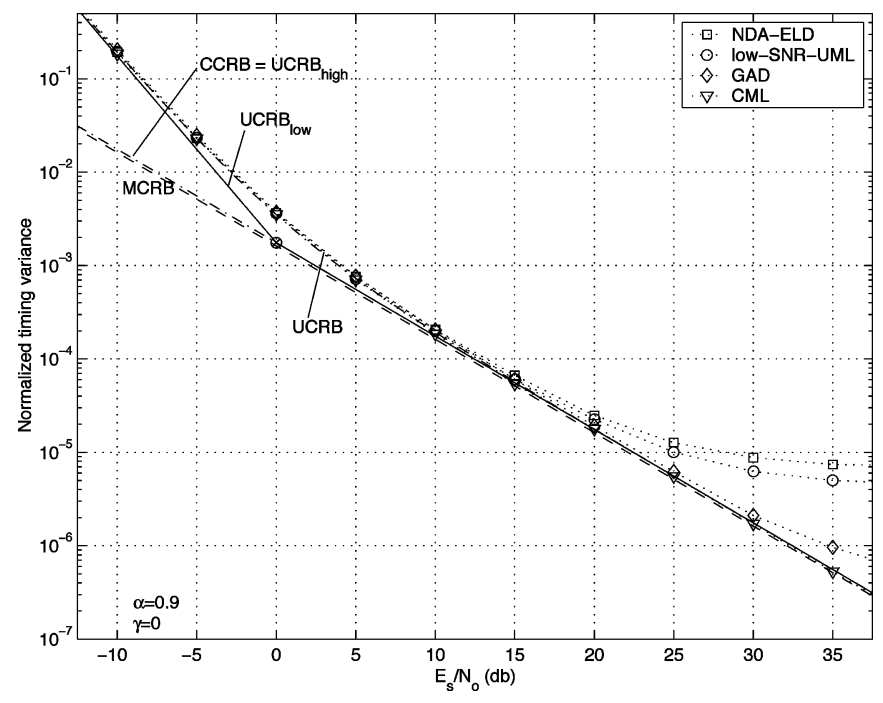

Fig. 9. Comparison of the performance of some quadratic timing recovery schemes with the new bounds for $L=64, \alpha=0.9$, and $\gamma=0$.

is smaller in that case. In Fig. 9, all the detectors attain the UCRB, except those affected by self noise, which exhibit a floor for high $E_{s} / N_{o}$. The MCRB is also depicted, which is very near the $\mathrm{UCRB}_{\text {high }}$ asymptote for roll-off factors near 1 . Fig. 10 confirms again the threshold effect predicted by the bound, which is a little smaller in that case, in comparison with that in Fig. 7. Finally, in the case of $100 \%$ transition density shown in Fig. 11, all detectors attain the UCRB at high $E_{s} / N_{o}$. However, for this case of alternated pattern and roll-off factor near 1 , it is seen that they depart more significantly from the UCRB at small $E_{s} / N_{o}$, which means that they have a threshold value of the $\left(E_{s} / N_{o}\right)$ th higher than that predicted by the bounds. With the purpose of validating the obtained result, an additional ad hoc quadratic timing detector has been considered in Fig. 11, which has been named high transition density NDA-ELD (HTD-NDA-ELD). Basically, it has the same form as the NDA-ELD but extending the length of the matched filter to various symbols taking into account the alternated pattern structure. It is important to note that although we are exploiting the data pattern structure, this is not a data-aided method, because it is still phase-independent and still quadratic with respect to the data, accordingly. In the simulations, a modified matched filter length of 12 symbols has been considered. It is shown in Fig. 11 that this simple estimator is able to yield a better performance at very low $E_{s} / N_{o}$ (very near the UCRB), just where the classical estimators are operating below their inherent threshold. It is noted that the HTD-NDA-ELD detector is the only one (among those presented here) that is designed, taking into account the presence of correlation among the symbols. These results tell us that the consideration of the data structure for the design of quadratic timing estimators is only necessary at very small $E_{s} / N_{o}$, because all estimators (as seen in Fig. 11) are able to attain the UCRB for correlated data for moderate and high $E_{s} / N_{o}$ without necessity of any modification. Only for very low $E_{s} / N_{o}$ have we had to adapt the estimator structure to the actual symbol transition density conditions.

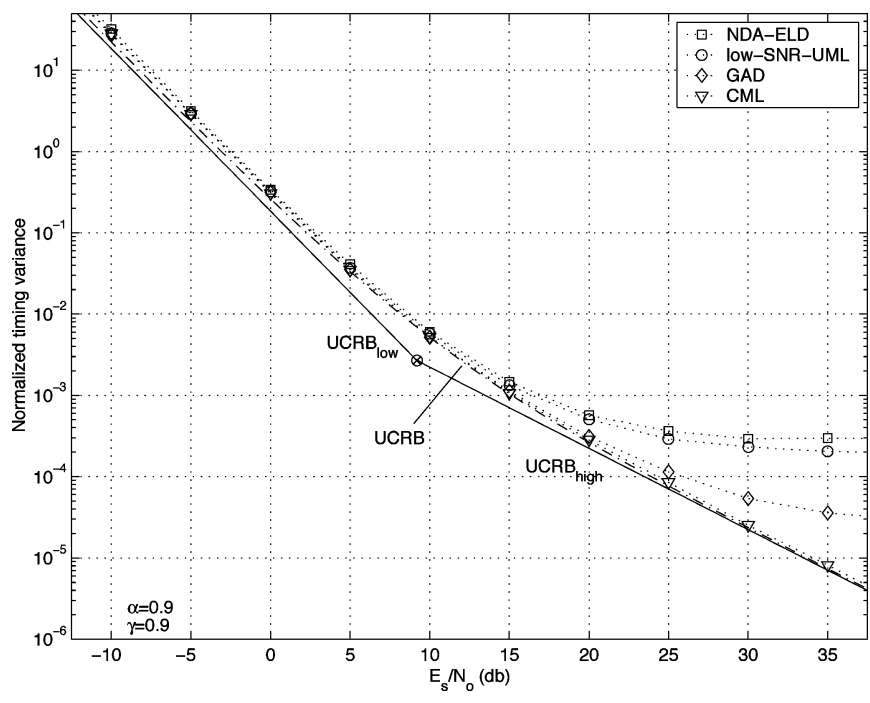

Fig. 10. Comparison of the performance of some quadratic timing recovery schemes with the new bounds for $L=64, \alpha=0.9$, and $\gamma=0.9$.

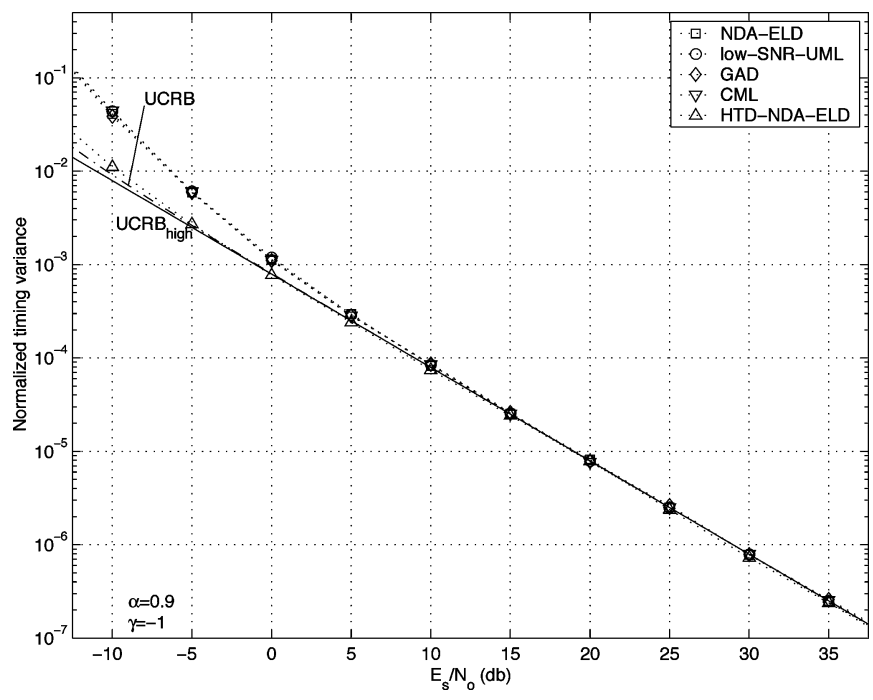

Fig. 11. Comparison of the performance of some quadratic timing recovery schemes with the new bounds for $L=64, \alpha=0.9$, and $\gamma=-1$.

\section{SUMMARY AND CONCLUSIONS}

In this paper, we have analyzed the effect of symbol transition density on the ultimate performance of quadratic NDA timing recovery schemes. Low and high approximations of the $\operatorname{UCRB}(\tau)$ can be computed through (22) (which generalizes [4] for correlated data) and (26), respectively. The coefficients $\xi$ for low and high SNR can be computed using (23) and (27), respectively, and they depend on both the pulse shape and the symbol correlation properties. Symbol correlation affects the coefficient $\xi$ through functions $q_{a}[p]$ and $r_{a}[p]$ in (32) and (30), respectively, which depend on the actual transition density present in the data symbols. The threshold $E_{s} / N_{O}$ can be easily computed using (28), and it represents the $E_{s} / N_{o}$, where the slope of the UCRB changes from -2 to $-1 \mathrm{~dB}$ per $\mathrm{dB}$. This threshold increases above $0 \mathrm{~dB}$ for low transition densities, whereas it decreases below $0 \mathrm{~dB}$ for high transition densities. This variation of the threshold is more significant 
for small roll-off factors. The UCRB admits a general expression for any $E_{s} / N_{o}$ given in (40) and (41), although the computation of the coefficient $\xi$ becomes more involved in this case. Equations (40) and (41) unify and generalize the work in [4] and [9] due to the following two facts: On the one hand, the present paper considers any degree of symbol correlation (not restricting only to the case of uncorrelated data as in [9]), and on the other hand, [4] is obtained as a particular case starting from a different perspective. Finally, the UCRB for fully correlated data (maximum and minimum transition densities) is given in (46) and (47). The threshold $E_{s} / N_{o}$ in these conditions becomes inversely proportional to the observation window $L$. Well-known quadratic timing error detectors are shown to attain the derived bound, concluding that no specialized estimator need to be designed for the case of symbol correlation, except in the case of high transition densities at very low $E_{s} / N_{o}$ (especially in the case of high excess bandwidths).

Future work will focus on the derivation of analytical expressions of the coefficients $\xi_{\text {low }}, \xi_{\text {high }}$, and $\xi$, as a function of $\alpha$ and $\gamma$. The research will also focus on the application of the derived bounds to a larger class of pulses, as those having built-in synchronization capabilities (as for instance the return-to-zero, RZ, and Manchester formats, [7], [17]), which are specifically designed to aid the timing synchronization. Preliminary analysis is showing that these kind of pulses yield a non-null coefficient $\xi_{\text {full }}$, even for the constant pattern $(\gamma=1)$, and then, (46) can be used as a benchmark in applications where very low transition densities can be expected.

\section{APPENDIX A}

In this Appendix, we prove that

$\mathbf{S}=\boldsymbol{\Gamma} \mathbf{A}_{\tau}^{T}\left(\sigma^{2} \mathbf{I}+\mathbf{A}_{\tau} \boldsymbol{\Gamma} \mathbf{A}_{\tau}^{T}\right)^{-1} \mathbf{A}_{\tau} \boldsymbol{\Gamma}=\boldsymbol{\Gamma}\left(\boldsymbol{\Gamma}+\sigma^{2}\left(\mathbf{A}_{\tau}^{T} \mathbf{A}_{\tau}\right)^{-1}\right)^{-1} \boldsymbol{\Gamma}$.

Applying the matrix inversion lemma to $\left(\sigma^{2} \mathbf{I}+\mathbf{A}_{\tau} \Gamma \mathbf{A}_{\tau}^{T}\right)^{-1}$, we get

$$
\sigma^{-2} \mathbf{I}-\sigma^{-2} \mathbf{A}_{\tau}\left(\sigma^{-2} \mathbf{A}_{\tau}^{T} \mathbf{A}_{\tau}+\boldsymbol{\Gamma}^{-1}\right)^{-1} \mathbf{A}_{\tau}^{T} \sigma^{-2}
$$

and applying it once more to $\left(\sigma^{-2} \mathbf{A}_{\tau}^{T} \mathbf{A}_{\tau}+\Gamma^{-1}\right)^{-1}$, we get

$$
\begin{aligned}
\sigma^{2}\left(\mathbf{A}_{\tau}^{T} \mathbf{A}_{\tau}\right)^{-1} & -\sigma^{2}\left(\mathbf{A}_{\tau}^{T} \mathbf{A}_{\tau}\right)^{-1} \\
& \times\left(\boldsymbol{\Gamma}+\sigma^{2}\left(\mathbf{A}_{\tau}^{T} \mathbf{A}_{\tau}\right)^{-1}\right)^{-1}\left(\mathbf{A}_{\tau}^{T} \mathbf{A}_{\tau}\right)^{-1} \sigma^{-2} .
\end{aligned}
$$

Substituting (50) in (49), we can write $\left(\sigma^{2} \mathbf{I}+\mathbf{A}_{\tau} \boldsymbol{\Gamma} \mathbf{A}_{\tau}^{T}\right)^{-1}$ as

$$
\begin{aligned}
\sigma^{-2} \mathbf{I}-\mathbf{A}_{\tau} & \left(\sigma^{-2}\left(\mathbf{A}_{\tau}^{T} \mathbf{A}_{\tau}\right)^{-1}-\left(\mathbf{A}_{\tau}^{T} \mathbf{A}_{\tau}\right)^{-1}\right. \\
& \left.\times\left(\boldsymbol{\Gamma}+\sigma^{2}\left(\mathbf{A}_{\tau}^{T} \mathbf{A}_{\tau}\right)^{-1}\right)^{-1}\left(\mathbf{A}_{\tau}^{T} \mathbf{A}_{\tau}\right)^{-1}\right) \mathbf{A}_{\tau}^{T}
\end{aligned}
$$

and inserting (51) in (48), we finally get

$$
\begin{aligned}
\mathbf{S} & =\boldsymbol{\Gamma}\left(\sigma^{-2} \mathbf{A}_{\tau}^{T} \mathbf{A}_{\tau}-\left(\sigma^{-2} \mathbf{A}_{\tau}^{T} \mathbf{A}_{\tau}-\left(\boldsymbol{\Gamma}+\sigma^{2}\left(\mathbf{A}_{\tau}^{T} \mathbf{A}_{\tau}\right)^{-1}\right)^{-1}\right)\right) \boldsymbol{\Gamma} \\
& =\boldsymbol{\Gamma}\left(\boldsymbol{\Gamma}+\sigma^{2}\left(\mathbf{A}_{\tau}^{T} \mathbf{A}_{\tau}\right)^{-1}\right)^{-1} \boldsymbol{\Gamma}
\end{aligned}
$$

as we wanted to prove.

\section{APPENDIX B}

In this Appendix, we prove that for $|\gamma|=1$ [see definition of $\gamma$ in (30)]

$$
\boldsymbol{\Gamma}\left(\boldsymbol{\Gamma}+\sigma^{2}\left(\mathbf{A}_{\tau}^{T} \mathbf{A}_{\tau}\right)^{-1}\right)^{-1} \boldsymbol{\Gamma}=\boldsymbol{\Gamma} \frac{\frac{L E_{s}}{N_{o}}}{1+\frac{L E_{s}}{N_{o}}}
$$

Using (43) and (3) in the second term of (48) and taking into account that $\sigma^{2} T_{s} / E_{g}=\sigma_{a}^{2}\left(E_{s} / N_{o}\right)^{-1}$, we have

$$
\begin{aligned}
\boldsymbol{\Gamma}\left(\boldsymbol{\Gamma}+\sigma^{2}\left(\mathbf{A}_{\tau}^{T} \mathbf{A}_{\tau}\right)^{-1}\right)^{-1} \boldsymbol{\Gamma} \\
=\sigma_{a}^{2} \mathbf{u} \mathbf{u}^{T}\left(\left(\frac{E_{s}}{N_{o}}\right)^{-1} \mathbf{I}+\mathbf{u u}^{T}\right)^{-1} \mathbf{u} \mathbf{u}^{T}
\end{aligned}
$$

Applying the matrix inversion lemma to the previous inverse, we obtain

$$
\sigma_{a}^{2} \frac{E_{s}}{N_{o}} \mathbf{u} \mathbf{u}^{T}\left(1-\frac{\frac{\mathbf{u}^{T} \mathbf{u} E_{s}}{N_{o}}}{1+\frac{\mathbf{u}^{T} \mathbf{u} E_{s}}{N_{o}}}\right) \mathbf{u u}^{T} .
$$

Taking into account that $\mathbf{u}^{T} \mathbf{u}=L$, we get

$$
\frac{\sigma_{a}^{2} L E_{s}}{N_{o}} \mathbf{u}\left(1-\frac{\frac{L E_{s}}{N_{o}}}{1+\frac{L E_{s}}{N_{o}}}\right) \mathbf{u}^{T}
$$

and considering again (43), we obtain

$$
\Gamma \frac{\frac{L E_{s}}{N_{o}}}{1+\frac{L E_{s}}{N_{o}}}
$$

as we wanted to prove.

\section{ACKNOWLEDGMENT}

The author like to thank constructive comments pointed out by M. Moeneclaey and anonymous reviewers, which helped in understanding the relationship of this contribution with previous work on digital synchronization.

\section{REFERENCES}

[1] A. D'Andrea, U. Mengali, and R. Reggiannini, "The modified Cramer-Rao bound and its application to synchronization problems," IEEE Trans. Commun., vol. 42, pp. 1391-1399, Feb./Mar./Apr. 1994.

[2] M. Moeneclaey, "A simple lower bound on the linearized performance of practical symbol synchronizers," IEEE Trans. Commun., vol. COM-31, pp. 1029-1032, Sept. 1983.

[3] — "On the true and the modified Cramer-Rao bounds for the estimation of a scalar parameter in the presence of nuisance parameters," IEEE Trans. Commun., vol. 46, pp. 1536-1544, Nov. 1998.

[4] H. Steendam and M. Moeneclaey, "Low-SNR limit of the Cramer-Rao bound for estimating the time delay of a PSK, QAM, or PAM waveform,' IEEE Commun. Lett., vol. 5, pp. 31-33, Jan. 2001.

[5] N. Noels, H. Steendam, and M. Moeneclaey, "The true Cramer-Rao bound for estimating the time delay of a linearly modulated waveform," in Proc. IEEE Int. Conf. Commun., vol. 3, 2002, pp. 1476-1479.

[6] C.-S. Tsang and C. M. Chie, "Effect of signal transition variation on bit synchronizer performance," IEEE Trans. Commun., vol. 41, pp. 673-677, May 1993.

[7] M. Moeneclaey, "Synchronizability of a general class of PCM formats, including NRZ, Manchester, and Miller coding," IEEE Trans. Commun., vol. 32, pp. 1020-1024, Sept. 1984. 
[8] B. Ottersten, M. Viberg, and P. Stoica, Radar Array Processing. New York: Springer-Verlag, 1993, ch. 4: Exact and Large Sample Maximum Likelihood Techniques for Parameter Estimation and Detection.

[9] G. Vazquez and J. Riba, Signal Processing Advances in Wireless and Mobile Communications, G. B. Giannakis, Y. Hua, P. Stoica, and L. Tong, Eds. Englewood Cliffs, NJ: Prentice-Hall, July 2000, vol. 2, Trends in Single- and Multi-User Systems, ch. 9: Non-Data-Aided Digital Synchronization.

[10] J. Riba, J. Sala, and G. Vazquez, "Conditional maximum likelihood timing recovery: estimators and bounds," IEEE Trans. Signal Processing, vol. 49, pp. 835-850, Apr. 2001.

[11] P. Stoica and A. Nehorai, "Performance study of conditional and unconditional direction-of-arrival estimation," IEEE Trans. Acoust., Speech, Signal Processing, vol. 38, pp. 1783-1795, Oct. 1990.

[12] B. Ottersten, M. Viberg, and T. Kailath, "Analysis of subspace fitting and ML techniques for parameter estimation from sensor array data," IEEE Trans. Signal Processing, vol. 40, pp. 590-600, Mar. 1992.

[13] H. Meyr, M. Moeneclaey, and S. A. Fechtel, Digital Communication Receivers. New York: Wiley, 1998.

[14] U. Mengali and A. D'Andrea, Synchronization Techniques for Digital Receivers. New York: Plenum, 1997.

[15] M. Moeneclaey, "A comparison of two types of symbol synchronizers for which self-noise is absent," IEEE Trans. Commun., vol. 31, pp. 329-334, Mar. 1983.
[16] G. Bilardi, R. Padovani, and G. L. Pierobon, "Spectral analysis of functions of Markov chains with applications," IEEE Trans. Commun., vol. COM-31, pp. 853-861, July 1983.

[17] S. Haykin, Digital Communications. New York: Wiley, 1988.

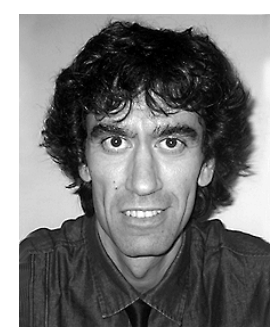

Jaume Riba was born in Barcelona, Spain, in 1966. He received the M.Sc. and Ph.D. degrees in telecommunications engineering from the Polytechnic University of Catalonia (UPC), Barcelona, Spain, in 1992 and 1997, respectively.

In 1992, he joined the Department of Signal Theory and Communications (TSC) and the Communications Signal Processing Group (GPS), UPC, as Assistant Professor and was promoted to Associate Professor in 1997. His current research interests are in the area of signal processing, communications, and wireless location, with particular emphasis on array processing and digital synchronization techniques. He has been involved in several signal processing research and development projects in the framework of the European Space Agency research programs, and he is a partner of the European Mobile Integrated Location sYstem (EMILY) project of the IST.

Dr. Riba received the 2003 Best Paper Award from the IEEE Signal Processing Society. 